\title{
S-Homocysteinylation effects on transthyretin: worsening of cardiomyopathy onset
}

\author{
Manuela Leri ${ }^{\mathrm{a}, \mathrm{b}}$, Paola Rebuzzini ${ }^{\mathrm{c}, \mathrm{d}}$, Anna Caselli ${ }^{\mathrm{a}}$, Simone Luti ${ }^{\mathrm{a}}$, Antonino Natalello ${ }^{\mathrm{e}}$, \\ Sofia Giorgettif ${ }^{\mathrm{f}}$ Loredana Marchese ${ }^{\mathrm{f}}$, Silvia Garagna ${ }^{\mathrm{c}, \mathrm{d}}$, Massimo Stefani ${ }^{\mathrm{a}, \mathrm{g}}$, Paolo Paoli ${ }^{\mathrm{a}}$, \\ Monica Bucciantini ${ }^{\mathrm{a}, \mathrm{g}, *}$ \\ ${ }^{a}$ Department of Experimental and Clinical Biomedical Sciences 'Mario Serio', University of Florence, Viale Morgagni 50, 50134 Florence, Italy \\ ${ }^{\mathrm{b}}$ Department of Neuroscience, Psychology, Area of Medicine and Health of the Child of the University of Florence, Viale Pieraccini, 6, 50139 Florence, Italy \\ ${ }^{\mathrm{c}}$ Department of Biology and Biotechnology, University of Pavia, Pavia, Italy \\ ${ }^{\mathrm{d}}$ Centre for Health Technologies (C.H.T.), Università degli Studi di Pavia, Pavia, Italy \\ ${ }^{\mathrm{e}}$ Department of Biotechnology and Biosciences, University of Milano Bicocca, Piazza della Scienza 2, 20126 Milano, Italy \\ ${ }^{\mathrm{f}}$ Department of Molecular Medicine, Institute of Biochemistry, University of Pavia, Viale Taramelli 3/B, 27100 Pavia, Italy \\ ${ }^{\mathrm{g}}$ Interuniversity Centre for the Study of Neurodegenerative Diseases (CIMN), Florence, Italy
}

\section{A R T I C L E I N F O}

\section{Keywords:}

Cardiomiopathy

Amyloid disease

FAP

FAC

L55P-TTR

Homocysteine

\begin{abstract}
A B S T R A C T
Background: L-Homocysteine (Hcy) is a non-proteinogenic $\alpha$-amino acid synthesized from dietary methionine. In healthy humans, high Hcy levels are a risk factor for cardiovascular diseases, stroke and type 2 diabetes. A recent study reports that Hcy reacts with $\mathrm{Cys}^{10}$ of transthyretin (TTR), generating a stable covalent adduct. However, to date the effect of S-homocysteinylation on TTR conformational stability remains unknown.

Methods: The effect of Hcy on the conformational properties of wt- and L55P-TTR were analysed using a set of biophysical techniques. The cytotoxicity of S-homocysteinylated L55P-TTR was also evaluated in the HL-1 cardiomyocyte cell line, while the effects of the assemblies on kinematic and dynamics properties of cardiac muscle cells were analysed in cardiomyocyte syncytia.

Results: We found that Hcy stabilizes tetrameric wt-TTR, while it destabilizes the tetrameric structure of the L55P mutant, promoting the accumulation of self-assembly-prone monomeric species.

Conclusions: Our study demonstrated that S-homocysteinylation of the L55P-TTR mutant impairs protein stability, favouring the appearance of toxic monomers. Interestingly, S-homocysteinylation affected only mutant, not wt-TTR. Moreover, we also show that assemblies of S-homocysteinylated L55P-TTR impair cardiomyocytes functional parameters.

General significance: Our study offers new insights on the negative impact of S-homocysteinylation on L55P-TTR stability, whose aggregation is considered the causative agent of a form of early-onset familial amyloid polyneuropathy and cardiomyopathy. Our results suggest that high homocysteine levels are a further risk factor for TTR cardiomyopathy in patients harbouring the L55P-TTR mutation.
\end{abstract}

\section{Introduction}

Hyperhomocysteinemia is a risk factor for cardiovascular disease and neurodegenerative disorders [1]. In the body fluids, free homocysteine (Hcy) accounts for only $2 \%$ of total Hcy while about $70-80 \%$ of Hcy is covalently bound to proteins. The remaining $20 \%$ of Hcy exists as both low-molecular-weight disulfides (homocystine) and Hcy-cysteine mixed disulfides [2-4]. Hcy binds covalently to free protein cysteine residues or cleaves accessible cysteine disulfide bonds (S-homocysteinylation) altering protein molecular structure and/or function $[4,5]$. Recently, several $S$-homocysteinylated proteins have been identified and the functional consequences of this covalent modification are starting to emerge. It has been proposed that protein homocysteinylation, rather than free Hcy, triggers cell responses related to up-regulation of effectors involved in vascular remodelling and in the pathogenesis of atherosclerotic lesions [6]. For instance, an in vitro study

\footnotetext{
Abbreviations: TTR, Transthyretin; Hcy, L-Homocysteine; wt-TTR-S-Hcy, S-homocysteynilated wt-TTR; L55P-TTR-S-Hcy, S-homocysteynilated L55P-TTR * Corresponding author at: Department of Experimental and Clinical Biomedical Sciences 'Mario Serio', University of Florence, Viale Morgagni 50, 50134 Florence, Italy.

E-mail address: monica.bucciantini@unifi.it (M. Bucciantini).
} 
reported that annexin II S-homocysteinylation at $\mathrm{Cys}^{8}$, hinders the specific interaction between tissue plasminogen activator and endothelial cell surface [7]. Moreover, it has been reported that homocysteinylation impairs inactivation of factor Va by activated protein $\mathrm{C}$ [8] and that the activation by Hcy of latent elastolytic metalloproteinase pro-MMP-2 could be one of the initial events that contribute to elastin alteration in patients affected by hyperhomocysteinemia [9]. Other findings indicate that Hcy in vitro interacts with fibronectin, thereby inhibiting its binding to fibrin [10], and that apolipoprotein homocysteinylation results in antiangiogenic and pro-atherogenic effects [11]. Finally, it has been reported that $S$-homocysteinylation causes the inactivation of metallothionein, a ubiquitous intracellular zinc-chaperone and superoxide anion radical scavenger, suggesting a novel mechanism for disruption of intracellular zinc and redox homeostasis [12]. Taken together, these findings suggest that protein homocysteinylation may affect multiple cellular pathways with important functional consequences.

Transthyretin (prealbumin) is a $55.2 \mathrm{kDa}$ protein synthesized predominantly in the liver and choroid plexi and secreted into the plasma and CSF, respectively. As a homotetramer, transthyretin binds and transports thyroxine and the retinol-binding protein-retinal complex [13]. Familial amyloid polyneuropathy/cardiomyopathy (FAP/FAC) and senile systemic amyloidosis (SSA) are pathological conditions associated to the extracellular deposition of amyloid aggregates of mutant or wild type TTR, respectively, even though SSA in some cases may also be genetically determined $[14,15]$. Polyneuropathy and cardiomyopathy are predominant signs of most TTR amyloidosis, whose most severe cases can be treated only by liver and, possibly, heart transplantation.

Generally, the most severe early onset TTR amyloidosis are associated to TTR variants (V122I, V30G, and L55P) whose tetrameric native state is remarkably destabilized. In these cases, mutation favours tetramer dissociation and the accumulation of monomers/dimers selfassociating into amyloid intermediates and mature fibrils [16]. Presently, TTR tetramer dissociation is recognized as the rate-limiting step of amyloid fibril nucleation and growth.

Each TTR monomer contains a single cysteine residue at position 10 $\left(\mathrm{Cys}^{10}\right)$. In the folded tetrameric protein, the four $\mathrm{Cys}^{10}$ residues occupy exposed sites at the beginning of the helical regions and can react with free cysteine and other sulfur-containing ligands [17], including Hcy [18]. Recently, the TTR-Cys ${ }^{10}-S-S-H c y$ adduct was identified by immunoprecipitation and HPLC/MS analysis in the plasma of patients with end-stage renal disease or affected by homocystinuria $[19,20]$. The TTR-Cys ${ }^{10}-$ S-S-Hcy/unmodified TTR ratio increases with increasing plasma concentration of Hcy, suggesting that an Hcy burden can affect the plasma levels of TTR-Cys ${ }^{10}-\mathrm{S}-\mathrm{S}-\mathrm{Hcy}$. To date, the functional, biochemical and pathological consequences of TTR S-homocysteinylation have not been completely assessed.

\section{Material and methods}

\subsection{TTR samples}

Recombinant wt-TTR and L55P-TTR were expressed and purified according to Mangione et al. [21,40]. Lyophilized wt-TTR and L55PTTR were dissolved at $1.6 \mathrm{mM}$ in $30 \mathrm{mM}$ sodium phosphate buffer, $\mathrm{pH}$ 7.0. wt-TTR and L55P-TTR were diluted at $50 \mu \mathrm{M}$ final concentration in the same buffer and incubated at $37^{\circ} \mathrm{C}$ for up to $96 \mathrm{~h}$ either alone or in the presence of Hcy in a 1:2 TTR tetramer: Hcy molar ratio.

\subsection{Growth of toxic HypF- $N$ oligomers}

HypF-N was expressed in E. coli and purified by an affinity chromatography column packed with the HIS-Select Nickel Affinity Gel (Sigma-Aldrich, St. Louis, MO, USA), as previously described [22,23]. The purified native protein was stored at $-80^{\circ} \mathrm{C}$ in $20 \mathrm{mM}$ Tris- $\mathrm{HCl}$ buffer, pH 8.0, containing $2.0 \mathrm{~mm}$ dithiothreitol (DTT) and used as a control (native HypF-N). Toxic HypF-N oligomers were grown by incubating the native protein $(48 \mu \mathrm{M})$ for $4 \mathrm{~h}$ at $25^{\circ} \mathrm{C}$ in $50 \mathrm{mM}$ acetate buffer, pH 5.5, containing $12 \%(\mathrm{v} / \mathrm{v})$ trifluoroethanol (TFE), $2.0 \mathrm{mM}$ DTT. The oligomers were collected by centrifugation at $16,000 \mathrm{~g}$ for $10 \mathrm{~min}$, dried under $\mathrm{N}_{2}$, and suspended to a final concentration of $12 \mu \mathrm{M}$ in cell culture medium for cell biology tests.

\subsection{TTR Digestion}

$20 \mu$ protein (wt-TTR; L55P-TTR; wt-TTR-S-Hcy; L55P-TTR-S-Hcy) were dissolved in $50 \mu \mathrm{L}$ of $50 \mathrm{mM}$ ammonium bicarbonate, $\mathrm{pH} 8.5$, containing $6.0 \mathrm{M}$ urea, incubated for $5 \mathrm{~min}$ in a boiling water bath and then chilled in ice. Then, $0,5 \mu \mathrm{g}$ of trypsin was added to the samples following dilution with $50 \mathrm{mM}$ ammonium bicarbonate, $\mathrm{pH} 8.5$ (5 vol.). After $2 \mathrm{~h}$ incubation at $37{ }^{\circ} \mathrm{C}$, the reaction was stopped by adding Trifluoroacetic acid (TFA) ( $0.1 \%$ final concentration). Then, the peptides were desalted and purified by using Zip-Tip C18, according to the manufacturer's protocol. To assess the right interpretation of the results, aliquots of each protein sample were reduced and alkylated prior to proteolytic digestion. Briefly, after the denaturation step, the proteins were treated with $20 \mathrm{mM}$ DTT at $60^{\circ} \mathrm{C}$. After $30 \mathrm{~min}$, Indoleacetic acid (IAA) (55 mM final concentration) was added; the samples were incubated at R.T. in the dark for $30 \mathrm{~min}$ and then added with DTT $(20 \mathrm{mM}$ final concentration).

\subsection{MS Analysis}

Peptide mass fingerprints were analysed with a MALDI-TOF/TOF mass spectrometer Ultraflex III (Bruker Daltonics, Bremen, Germany) by using Flex Control 3.0 as data acquisition software. A $0.75 \mu \mathrm{L}$ sample volume was mixed with $0.75 \mu \mathrm{L}$ of the matrix (saturated solution of $\alpha$ cyano-4-hydroxycinnamic acid in $50 \%(\mathrm{v} / \mathrm{v})$ acetonitrile and $0.5 \%(\mathrm{v} /$ v) TFA) on the anchorchip target plate and allowed to dry. The spectra were acquired in reflectron mode in the $860-4000 \mathrm{~m} / \mathrm{z}$ range, for 500 shots. The instrumental parameters were chosen by setting the ion source 1 at $25 \mathrm{kV}$, ion source 2 at $21.5 \mathrm{kV}$, the pulsed ion extraction at $20 \mathrm{~ns}$ and the detector gain at $7.7 \times$. The instrument was externally calibrated prior to analysis using the Bruker Peptide Calibration standard kit. The spectra were processed and annotated by the Flex Analysis 3,0 software. Peptide mass finger prints were interpreted using the BioTools 3,2 and the Sequence Editor software (Bruker Daltonics). Digestion results were compared with the theoretical ones obtained by virtual digestion of wt-TTR or L55P-TTR. The parameters used were: trypsin as cleaving enzyme, allowing three missed cleavages, and 50 p.p.m. mass tolerance. Reduced cysteine, carbamidomethyl cysteine (CAM-C) or homocysteinyl-cysteine (Hcys-C) was alternatively set as a fixed modification.

\subsection{SDS-PAGE}

Proteins were separated by SDS-PAGE. The samples were dissolved in Laemmli sample buffer (62.5 mM Tris-HCl buffer, pH 6.8, 2.0\% SDS, $25 \%$ glycerol, $0.01 \%$ bromphenol blue). $0.1 \% \quad \beta$-mercaptoethanol (BME) was added before sample boiling in a water bath $\left(10 \mathrm{~min}, 95^{\circ} \mathrm{C}\right)$. Then, $10 \mu \mathrm{L}$ samples were applied onto the $15 \%$ SDS-PAGE gel. The bands were visualized by Coomassie Brilliant Blue. SDS-PAGE was performed three times.

\subsection{Native-PAGE}

"Native" or "non-denaturing" gel electrophoresis was run in the absence of SDS. Running Buffer $5 \times$, Leammli $2 \times$ and $12 \%$ acrylamide gel without SDS were used. The samples were not boiled and were dissolved in Laemmli sample buffer without SDS. The bands were visualized by Coomassie Brilliant Blue. Native-PAGE was performed three 
times.

\subsection{Fourier transform infrared (FTIR) spectroscopy}

The FTIR spectra of the different samples were collected in attenuated total reflection (ATR) at a protein concentration of $20 \mu \mathrm{M}$ (in $30 \mathrm{mM}$ sodium phosphate buffer $\mathrm{pH} 7.0$ ) in the absence or in the presence of Hcy (1:2 ratio). In particular, $3 \mu \mathrm{L}$ sample aliquots were deposed on the ATR diamond plate (Golden Gate, Specac, UK) and the spectra were acquired after solvent evaporation, as previously described [24,25]. A Varian 670-IR spectrometer (Varian Australia) was employed under the following conditions: $2.0 \mathrm{~cm}^{-1}$ spectral resolution, $25 \mathrm{kHz}$ scan speed, 1000 scan coadditions, and triangular apodization. The second derivatives of the measured spectra were obtained after Savitsky-Golay smoothing by the Resolutions-Pro software (Varian Australia).

\subsection{Turbidimetry}

The propensity of L55P-TTR to aggregate was monitored by turbidimetry. The aggregation process was measured by spectrophotometry as the increase in the apparent absorbance value. Aggregate growth results in the formation of a cloudy suspension that scatters the light reducing its passage through the sample. According to the method previously reported [26] we monitored sample turbidity in a 96 multiwell plate containing 5.0 $\mathrm{MM}$ L55P-TTR alone or in the presence of $10 \mu \mathrm{M}$ Hcy (1:2 molar ratio) in sodium phosphate buffer, $\mathrm{pH} 7.0$, at $37^{\circ} \mathrm{C}$ without stirring. Sample absorbance was determined at $400 \mathrm{~nm}$ with an automatic plate reader (Bio-Rad).

\subsection{HPLC analysis}

HPLC analysis of wt-TTR and L55P-TTR samples incubated in the absence or in the presence of Hcy for $24 \mathrm{~h}$ was performed by a DIONEX P680-UVD170U system after centrifugation at $14000 \mathrm{rpm}$ for $30 \mathrm{~min}$ at $4{ }^{\circ} \mathrm{C}$. Sample chromatographic resolution was achieved on a $300 \times 7.80 \mathrm{~mm}, 5.0 \mu \mathrm{m}$, BioSep-SEC-S 3000 column (Phenomenex) using $100 \mathrm{mM}$ sodium chloride in $30 \mathrm{mM}$ phosphate buffer, $\mathrm{pH} 7.0$ at a flow rate of $0.5 \mathrm{~mL} / \mathrm{min}$. $50 \mu \mathrm{L}$ sample injection volume was used; sample detection was based on UV absorbance at $280 \mathrm{~nm}$.

\subsection{Transmission electron microscopy}

$5.0 \mu \mathrm{L}$ aliquots of L55P-TTR incubated in the absence or in the presence of Hcy were withdrawn at different incubation times, loaded onto a formvar/carbon-coated 400 mesh nickel grids (Agar Scientific, Stansted, UK) and negatively stained with $2.0 \%(\mathrm{w} / \mathrm{v})$ uranyl acetate (Sigma-Aldrich). The grid was air-dried and examined using a JEM 1010 transmission electron microscope at $80 \mathrm{kV}$ excitation voltage.

\subsection{Circular dichroism spectroscopy}

The spectra of wt-TTR $(5.0 \mu \mathrm{M}$ in $30 \mathrm{mM}$ sodium phosphate buffer $\mathrm{pH}$ 7.0) were recorded in the absence or in the presence of Hcy (1:2 ratio) using a Jasco J-810 spectropolarimeter equipped with a thermostated cell holder attached to a Thermo Haake C25P water bath. The spectra were acquired with a $2.0 \mathrm{~cm} / \mathrm{min}$ can speed and data points were collected in the $260-190 \mathrm{~nm}$ interval at $25^{\circ} \mathrm{C}$ with a $1.0 \mathrm{~mm}$ path length quartz cell. All spectra were blank-subtracted and converted to molar ellipticity per residue.

\subsection{Dynamic light scattering (DLS)}

DLS measurements were performed using a ZetasizerNano S DLS device from Malvern Instruments (Malvern, Worcestershire, UK), thermostated with a Peltier system using a low-volume $(45 \mu \mathrm{L})$ ultramicro cell (Hellma Analytics, Müllheim, Germany). wt-TTR and L55P-TTR incubated in the absence or in the presence of Hcy for different lengths of time, were centrifuged for $30 \mathrm{~min}$ at $4{ }^{\circ} \mathrm{C}$ and $14,000 \mathrm{rpm}$, to precipitate the largest aggregates. Size distributions by intensity and volume were determined at regular time-intervals over a period of $10 \mathrm{~min}$. The temperature was maintained at $37{ }^{\circ} \mathrm{C}$ and the parameters were set manually on the instrument to allow the same settings in the various distributions acquired at different times. These included ten $10 \mathrm{~s}$ acquisitions with cell position $4.2 \mathrm{~cm}$ and attenuator index 7. The reported data are the average of three consecutive measurements.

\subsection{Resveratrol binding assay}

The fluorescence studies were based on the method reported by Florio and co-workers [27]. Briefly, resveratrol was excited at $320 \mathrm{~nm}$ and the emission spectra were recorded in the $350-450 \mathrm{~nm}$ range. Free resveratrol displays maximum fluorescence at $390 \mathrm{~nm}$. Fluorescence intensity increased substantially upon binding to TTR. All data were obtained in the presence of $5.0 \mu \mathrm{M}$ wt-TTR and L55P-TTR in $30 \mathrm{mM}$ sodium phosphate buffer, pH 7.0 alone or pre-incubated with $10 \mu \mathrm{M}$ Hcy. At different incubation times, sample aliquots were withdrawn and mixed with $10 \mu \mathrm{M}$ resveratrol for analysis.

\subsection{Isolation and culture of $H L-1$ cardiomyocytes}

HL-1 mouse atrial myocytes were obtained from Dr. W. C. Claycomb (Louisiana State University Health Science Center, New Orleans, LA, USA) and grown in T25, gelatin- fibronectin coated flasks, as previously described [28]. The cells were maintained in Claycomb Medium (JRH Biosciences), supplemented with $10 \%$ foetal bovine serum (Sigma-Aldrich), $2.0 \mathrm{mM}$ L-glutamine (Sigma-Aldrich), $0.1 \mathrm{mM}$ noradrenaline (Sigma-Aldrich) and $100 \mathrm{U} / \mathrm{mL}$ penicillin-streptomycin (Sigma-Aldrich). Every three days the cells were detached and re-plated at a 1:3 dilution in a new T25 flask or in 96 well plates (70-90\% confluent) and used for experimental measurements.

\subsection{MTT assay}

The MTT assay, used to assess cell viability, was optimized for the cell lines used in the experiments. Briefly, HL-1 cells were seeded into 96-well plates at a density of 6000 cells/well in fresh complete medium and grown for $48 \mathrm{~h}$. Then, the cells were treated for $24 \mathrm{~h}$ with $5.0 \mu \mathrm{M}$ L55P-TTR (tetramer) at different times of aggregation in the presence or in the absence of Hcy $(10 \mu \mathrm{M})$. To analyse protection by L55P-TTR-SHcy, HypF-N oligomers (12 $\mu M$ monomer concentration) were shacked in the cell culture media for $1 \mathrm{~h}$ at $37^{\circ} \mathrm{C}$, either alone or in the presence of L55P-TTR or L55P-TTR-S-Hcy, at a HypF-N:TTR molar ratio of 10:1. L55P-TTR-S-Hcy was obtained by incubating the protein with or without Hcy for $30 \mathrm{~min}$ and $96 \mathrm{~h}$. Each sample was added to the cell culture medium for $24 \mathrm{~h}$; then, the medium was removed and the cells were incubated for $1 \mathrm{~h}$ at $37^{\circ} \mathrm{C}$ with $100 \mu \mathrm{L}$ of serum-free DMEM without phenol red, containing $0.5 \mathrm{mg} / \mathrm{mL}$ MTT. At the end of the incubation, $100 \mu \mathrm{L}$ of cell lysis solution ( $20 \%$ SDS, $50 \% \quad \mathrm{~N}, \mathrm{~N}$-dimethylformamide) was added to each well and the samples were incubated at $37^{\circ} \mathrm{C}$ to allow complete lysis. The absorbance of the blue formazan was read automatically at $570 \mathrm{~nm}$ using a spectrophotometric microplate reader. The final absorption values were calculated by averaging each sample in triplicate and subtracting from this the average of the blank, (a mixture of $100 \mu \mathrm{L}$ of MTT solution and $100 \mu \mathrm{L}$ of lysis solution).

\subsection{Intracellular calcium flux}

Briefly, HL-1 cells were cultured in an 8-well Lab-Tek II Chamber slide system (Thermo-Fisher) in complete medium containing 10\% FBS. After $48 \mathrm{~h}$, the cells were loaded for $10 \mathrm{~min}$ with FLUO-3 AM 

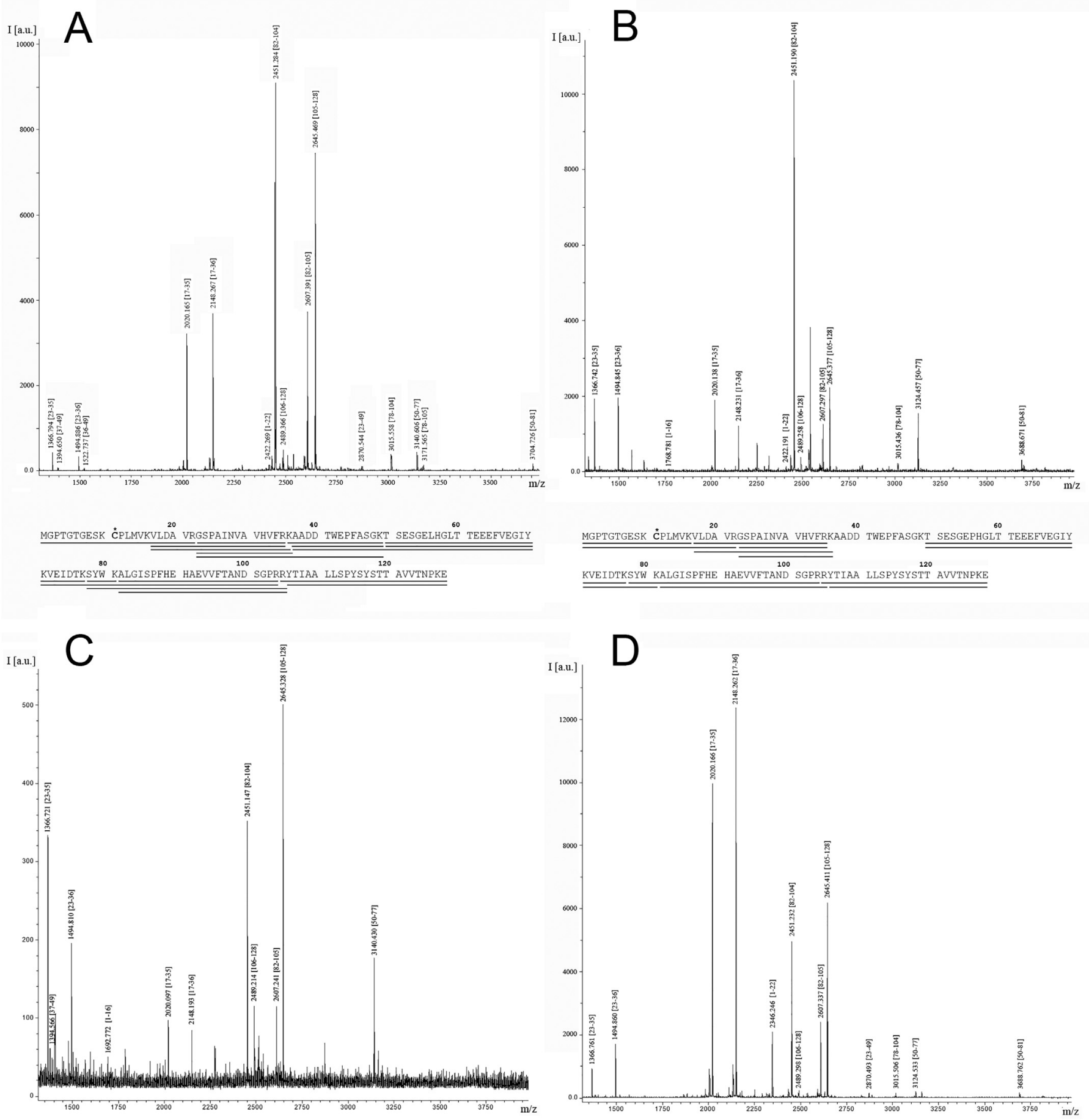

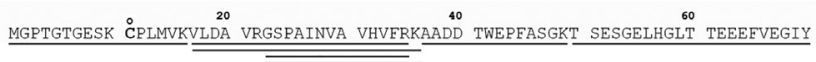
KVEIDTKSYW KALGISPFHE HAEVVETAND SGPRRYTIAA LLSPYSYSTT AVVTNPKE

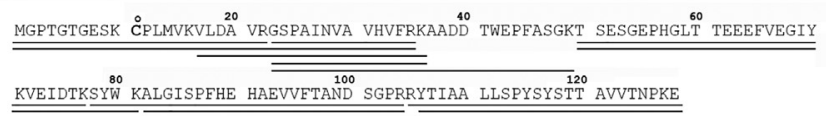

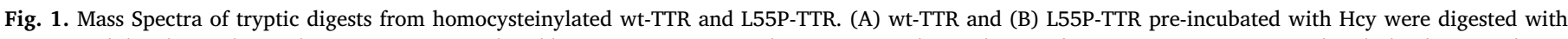

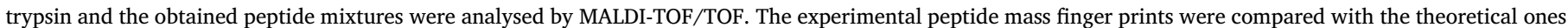

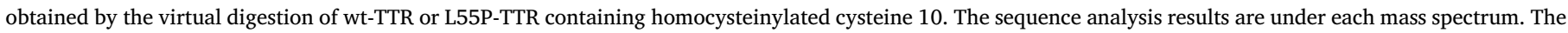

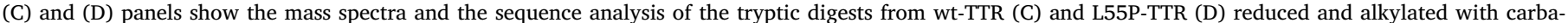
midomethyl-cysteine prior to digestion. * Homocysteinylated Cysteine; ${ }^{\circ}$ Carbamidomethylated Cysteine.

(Invitrogen, Inc.), a fluorescent cell $\mathrm{Ca}^{2+}$ indicator. Then, the L55P-TTR samples obtained by incubation for different times with Hcy (1:2 molar ratio) were added to the medium at $5.0 \mu \mathrm{M}$ final concentration. Cell fluorescence was imaged using a confocal Leica TCS SP5 scanning microscope (Leica, Mannheim, Ge) equipped with a $\mathrm{HeNe} / \mathrm{Ar}$ laser source for fluorescence measurements. The observations were performed using a Leica Plan 7 Apo X63 oil immersion objective, suited with optics for DIC acquisition. For image acquisition, an exposure time 
of $30 \mathrm{~s}$ was used for a total time of $5 \mathrm{~h}$. Cells from three independent experiments and three areas (about 20 cells/area) per experiment were analysed. The fluorescence intensity of Fluo3-AM was analysed by using ImageJ software (National Institutes of Health Bethesda, MD), and expressed as arbitrary units.

\subsection{Embryonic stem cell culture and differentiation into cardiomyocytes}

R1 mouse embryonic stem cell line (mESCs; kindly provided by Dr. Nagy from Samuel Lunenfeld Research Institute, Mount Sinai Hospital, Toronto, Ontario, Canada) was cultured as reported in [29]. Briefly, R1 mESCs were maintained in Knockout DMEM, added with 15\% ESC Qualified FBS (Gibco), $2.0 \mathrm{mM}$ L-glutamine, $1 \times$ non-essential amino acids, $0.5 \%$ penicillin/streptomycin (all from Thermo Fisher Scientific), $0.1 \mathrm{mM} \beta$-mercaptoethanol (Sigma) and $500 \mathrm{U} / \mathrm{mL}$ ESGRO-Leukemia Inhibitory Factor (LIF; Merck Millipore, Italy). The STO cell line (ATCC CRL-2225), used as feeder layer for mESCs, was maintained in DMEM (Sigma) supplemented with $10 \%$ foetal bovine serum, $4.0 \mathrm{mM}$ L-glutamine, $0.5 \%$ penicillin-streptomycin solution (all from Thermo Fisher Scientific), $0.1 \mathrm{mM} \beta$-mercaptoethanol (Sigma) and $0.2 \mathrm{mg} / \mathrm{mL}$ geneticin (Sigma). The cells were maintained at $37^{\circ} \mathrm{C}$ with $5 \% \mathrm{CO}_{2}$ in air. mESCs were induced to differentiate in vitro by removing the LIF from the culture medium and through the formation of embryoid bodies (EBs), using the hanging drop method [30,31]. For EBs formation, $20 \mu \mathrm{L}$ droplets of culture medium containing $10^{3}$ mESCs were plated on the lid of p55 Petri dish. On day 3 of culture, the developing EBs were transferred to $0.1 \%$ agarose-coated dishes (Corning) and from day 5 , about $25 \mathrm{EBs}$ were plated onto $22 \mathrm{~mm}$ gelatin-coated Glass Bottom Dishes (WillCo Wells) and cultivated up to day 15.

\subsection{Treatment with L55P-TTR and L55P-TTR-S-Hcy, video acquisition and processing for kinematic and dynamics properties evaluation}

At the end of the differentiation process (day 15), the cells were exposed for $24 \mathrm{~h}$ to $5.0 \mu \mathrm{M}$ L55P-TTR incubated $96 \mathrm{~h}$ in sodium phosphate buffer, pH 7.0, in the absence or in the presence of $10 \mu \mathrm{M}$ Hcy (1:2 molar ratio). On day 16 , the glass bottom dishes with beating cardiomyocyte syncytia were transferred into the culture chamber of the Nikon BioStation IM at $37^{\circ} \mathrm{C}$ and $5 \% \mathrm{CO}_{2}$. AVI videos were recorded from ten randomly chosen untreated or treated beating syncytia with the Snagit software and processed as previously described [32]. This procedure was repeated for three independent experiments.

\subsection{Statistical analysis}

Analysis of the data was performed by using T-student test to determine differences in cytotoxicity. The syncytium contractile properties are expressed as mean $\pm 95 \%$ confidence interval for the differences between means and the data were analysed by the one-way ANOVA and by the post hoc LSD test.

\section{Results}

Hcy levels are typically higher in men than in women and increase with age; normal serum levels of total Hcy are in the 4.0-15 $\mu \mathrm{M}$ range [33] and concentrations above $15 \mu \mathrm{M}$ are considered dangerous. Taking into account that only $2 \%$ of total Hcy is present in plasma as reduced form, we sought to test the effects of pathological concentration of soluble Hcy $(10 \mu \mathrm{M})$ on stability and aggregation propensity of wt-TTR and the highly destabilized mutant L55P-TTR.

\subsection{S-Homocysteinylation of wt-TTR and L55P-TTR}

Homocysteinylation of wt-TTR and L55P-TTR was obtained by incubating either protein for $24 \mathrm{~h}$ in the presence of $10 \mu \mathrm{M}$ Hcy; the extent of S-homocysteinylation was assessed by sample digestion with trypsin and subsequent analysis by MALDI-TOF (Fig. 1). The experimental $\left[\mathrm{M}+\mathrm{H}^{+}\right]$peak list generated for each protein was compared with the theoretical $\left[\mathrm{M}+\mathrm{H}^{+}\right]$peak list generated by "in silico" digestion of wtTTR and L55P-TTR, by setting Cys-S-homocysteinylation as variable modification. The results confirmed that the tryptic digests from both proteins contain peptides with a molecular weight compatible with that of Cys ${ }^{10}$-homocysteinylated peptides (Fig. 1A, B). In both cases, we did not identify the $\left[\mathrm{M}+\mathrm{H}^{+}\right]$peaks putatively derived from the unmodified (reduced) proteins nor disulfide bridge-containing peptides. To confirm the validity of our results, we also analysed the tryptic digests of the homocysteinylated proteins preliminarily reduced and alkylated with carbamidomethyl-cysteine (Fig. 1 C, D). The spectra missed the $\left[\mathrm{M}+\mathrm{H}^{+}\right]$homocysteinylated peptides signals while showed the $\left[\mathrm{M}+\mathrm{H}^{+}\right]$peaks corresponding to carbamidomethyl peptides.

\subsection{S-Homocysteinylation stabilizes the native conformation of wt-TTR}

The effect of S-homocysteinylation on the structural features of wtTTR (1:2; tetrameric wt-TTR:Hcy molar ratio) was analysed by nativeand SDS-PAGE, resveratrol binding, circular dichroism (CD), Fouriertransform infrared (FTIR) spectroscopy, gel filtration and dynamic light scattering (DLS). Electrophoretic analysis under native conditions showed that following incubation of wt-TTR with Hcy the relative amount of tetrameric TTR increased (Fig. 2A). Similar results were obtained analysing the same sample in a SDS-PAGE system. The wt-TTR sample displayed two bands (monomer and dimer) whose extent apparently decreased in the sample incubated with Hcy, with the appearance of a new, $55 \mathrm{kDa}$, band corresponding to the tetramer. Interestingly, we observed that the band corresponding to TTR tetramer disappeared in the presence of $0.1 \%$ BME, a reducing agent (Fig. 2B). This evidence suggested that reaction of Hcy with $\mathrm{Cys}^{10}$ residue of TTR favours stabilization of TTR tetramer, which was almost completely lost when Hcy was displaced by BME.

$t$-Resveratrol ( $t$-Res) is a polyphenol known to bind and to stabilize the wt-TTR tetramer. We evaluated $t$-Res binding to wt-TTR using a spectrofluorimeter. When excited at $320 \mathrm{~nm}, t$-Res displays a specific emission spectrum with a maximum fluorescence peak around $390 \mathrm{~nm}$ that is enhanced upon binding to wt-TTR tetramer [27]. Using this binding assay, we observed that S-homocysteinylated wt-TTR maintained the same ability to bind $t$-Res as the unmodified protein, suggesting that S-homocysteinylation did not alter the polyphenol binding to wt TTR (Fig. 2C).

We next investigated whether TTR S-homocysteinylation altered the size and the structure of tetrameric TTR. DLS analysis revealed that both wt-TTR and wt-TTR-S-Hcy incubated at pH 7.0 up to $24 \mathrm{~h}$ showed an apparent hydrodynamic diameter $\left(\mathrm{D}_{\mathrm{h}}\right)$ of about $6.0 \mathrm{~nm}$, consistent with that of the tetrameric structure of native TTR (Fig. 2D) [34]. Moreover, CD analysis did not reveal any significant change of secondary structure in homocysteinylated wt-TTR (Fig. 2E). The content of secondary structure in wt-TTR and wt-TTR-S-Hcy was also assessed by Amide $I$ band analysis $\left(1700-1600 \mathrm{~cm}^{-1}\right)$ in the protein FTIR spectra (Fig. 2F). As previously reported [24,25], the second derivative of the absorption spectrum of wt-TTR was dominated by a component around $1630 \mathrm{~cm}^{-1}$, assigned to native $\beta$-sheet structure (Fig. 2F). Additional components were also observed in the spectrum, whose assignments to protein native secondary structures are indicated in Fig. 2F [24,25]. No significant conformational changes were detected in wt-TTR-S-Hcy by FTIR spectroscopy (Fig. 2F). Finally, we did not detect any difference between wt-TTR and wt-TTR-S-Hcy retention times in gel filtration analysis (Fig. 2G). Taken together, our biophysical data suggest that Shomocysteinylation does not induce any structural modification of the tetrameric fold in wt-TTR as confirmed also by SDS-PAGE of wt-TTR incubated with Hcy for $96 \mathrm{~h}$ (Fig. 2H). 

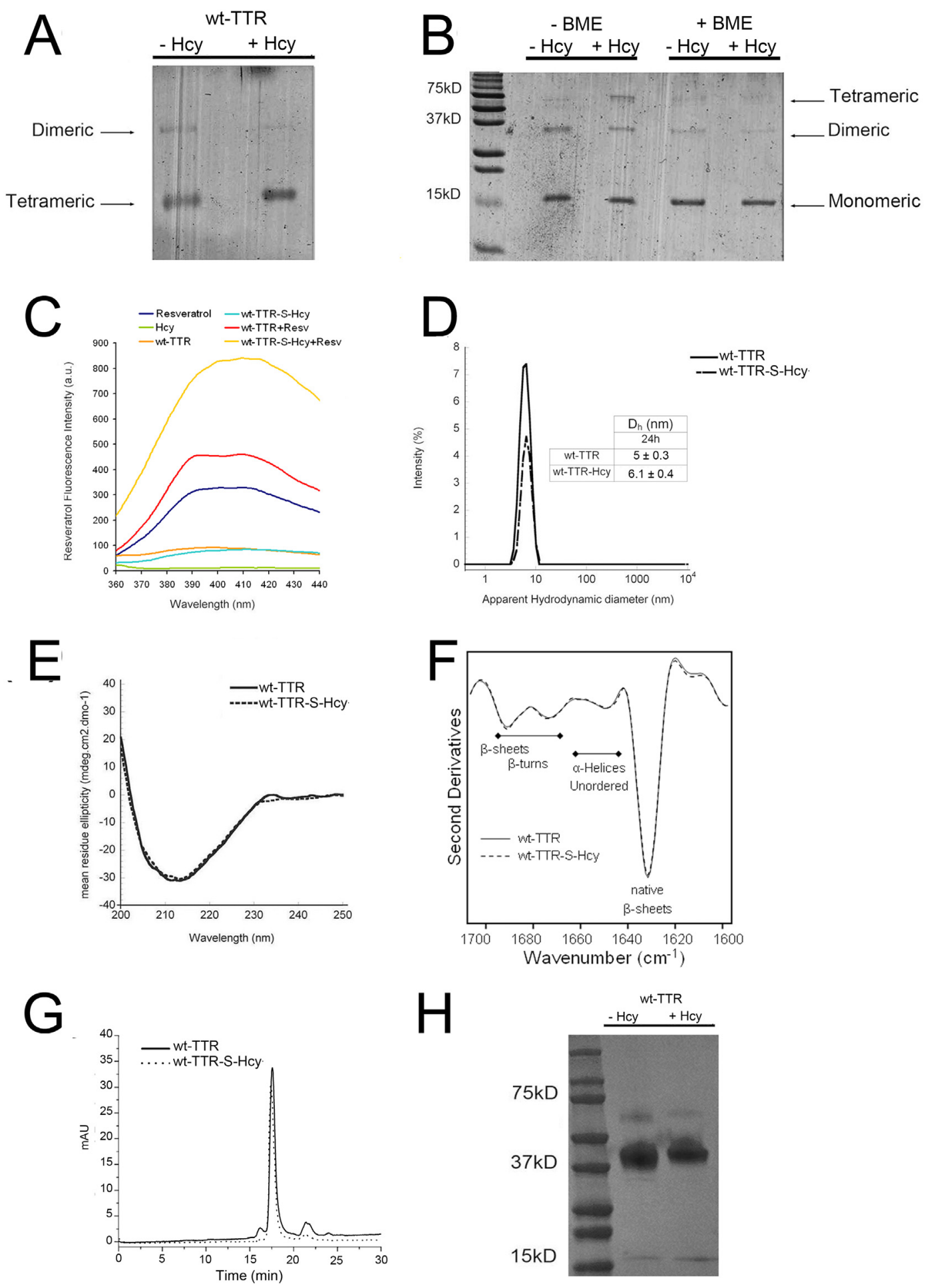

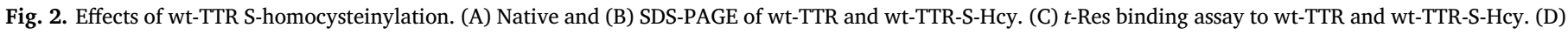

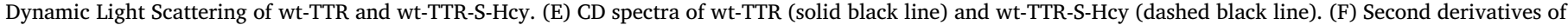

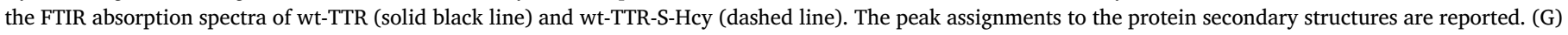

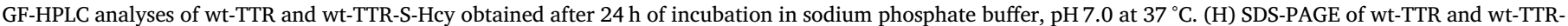
S-Hcy after $96 \mathrm{~h}$ of incubation with Hcy. The data shown are representative of three independent experiments.

\subsection{S-Homocysteinylation destabilizes the L55P-TTR tetrameric fold}

Based on results reported above, we wondered whether S-homocysteinylation produced similar effects on the L55P-TTR mutant. We preliminarily showed by mass spectrometry the presence of a disulphide bridge between Hcy and the $\mathrm{Cys}^{10}$ residue of L55P-TTR
(Fig. 1B, D). When we checked the ability to interact with $t$-Res, we found that, contrary to wt-TTR-S-Hcy, L55P-TTR-S-Hcy did not bind the polyphenol (Fig. 3A), indicating destabilization of the tetrameric structure even after $30 \mathrm{~min}$ of incubation with Hcy. Moreover, gel filtration analysis showed that the soluble protein content was significantly reduced in samples containing L55P-TTR and Hcy, suggesting 
A

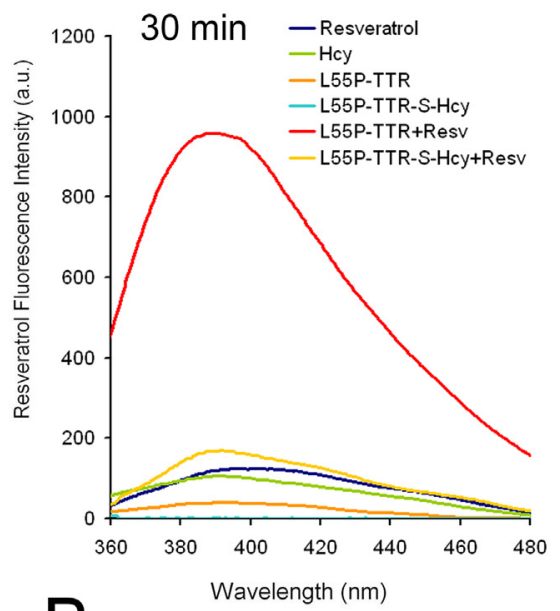

B

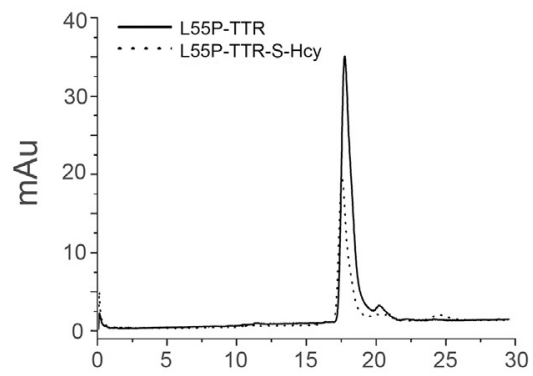

D

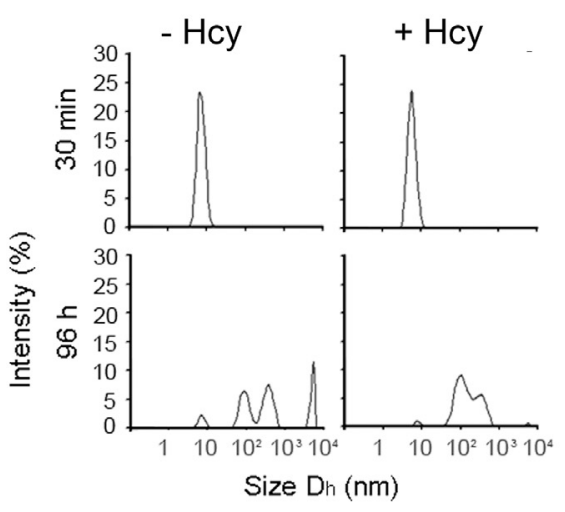

$\mathrm{F}$

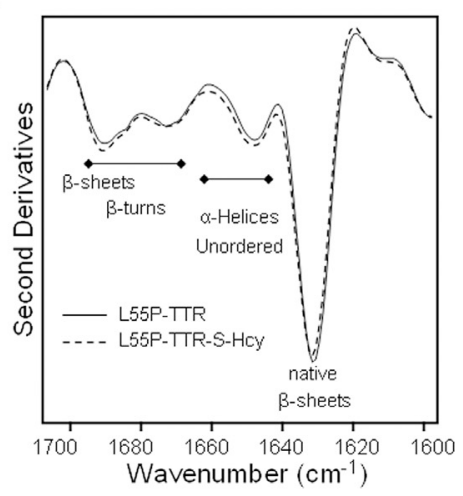

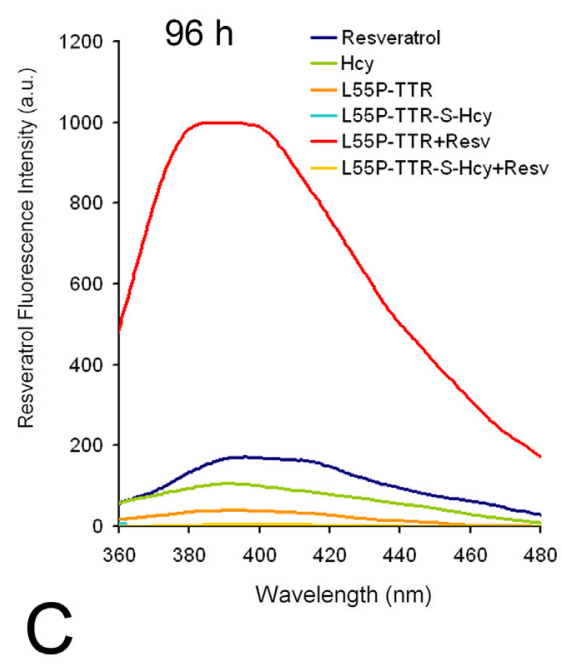

\begin{tabular}{|c|c|c|}
\cline { 2 - 3 } \multicolumn{1}{c|}{} & \multicolumn{2}{c|}{$\mathrm{D}_{\mathrm{h}}(\mathrm{nm})$} \\
\cline { 2 - 3 } \multicolumn{1}{c|}{} & $30 \mathrm{~min}$ & $96 \mathrm{~h}$ \\
\hline \multirow{3}{*}{ L55P-TTR } & $6.6 \pm 0.2$ & $6.5 \pm 0.2$ \\
& & \\
\hline L55P-TTR-S-Hcy & $6.9 \pm 0.3$ & $6 \pm 0.3$ \\
& & 91.28 \\
& & 500.78 \\
\hline
\end{tabular}

$E$

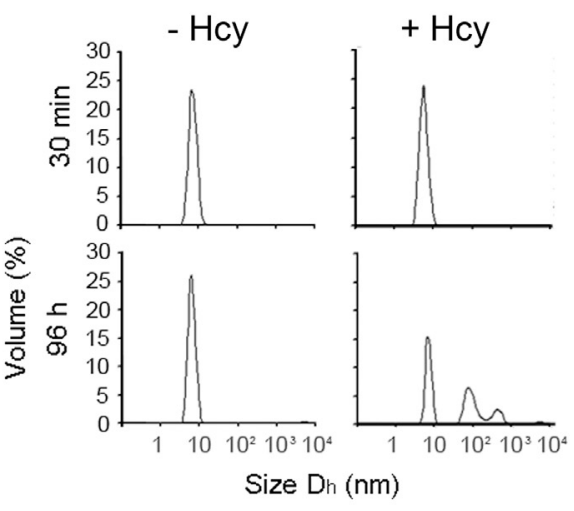

G

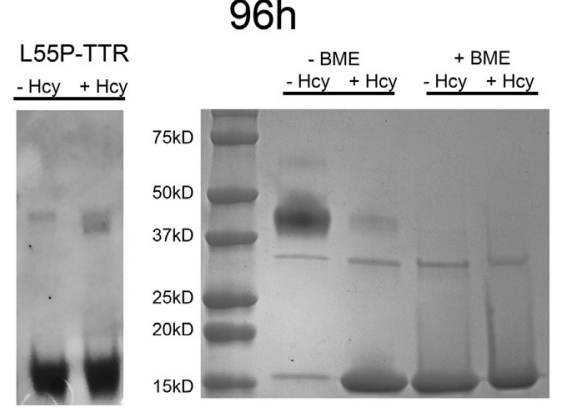

Fig. 3. Size distribution and resveratrol binding assay of L55P-TTR and L55P-TTR-S-Hcy. (A) Resveratrol binding assay of L55P-TTR and L55P-TTR-S-Hcy at pH7.0 at the two different incubation times. (B) GF-HPLC analyses of L55PTTR and L55P-TTR-S-Hcy obtained by incubation for $24 \mathrm{~h}$ in sodium phosphate buffer, $\mathrm{pH} 7.0$, at $37^{\circ} \mathrm{C}$. (C) Apparent Hydrodynamic diameter values observed in the same samples analysed by DLS at two different incubation times Size distribution over time of L55P-TTR and L55PTTR-S-Hcy at pH 7.0 as shown by DLS intensity spectra (D) and volume spectra (E). (F) Second derivatives of the absorption spectra of L55PTTR (solid black line) and of L55P-TTR-S-Hcy (dashed line). The peak assignments to the protein secondary structures are reported. (G) Native-PAGE (left) and SDS-PAGE (right) L55PTTR and of L55P-TTR-S-Hcy after $96 \mathrm{~h}$ of incubation without and with Hcy, respectively. The data shown are representative of three independent experiments. 
that L55P-TTR is destabilized by Hcy (Fig. 3B). DLS analysis confirmed this hypothesis. In fact, we found that $30 \mathrm{~min}$ incubation of L55P-TTR with Hcy resulted in a population of particles with $D_{h}$ of about $6.0 \mathrm{~nm}$, a value consistent with the apparent hydrodynamic diameter of the tetrameric protein (Fig. 3C-E). However, after $96 \mathrm{~h}$ of incubation, L55PTTR appeared still homogeneously composed of particles of about $6.0 \mathrm{~nm} \mathrm{D}_{\mathrm{h}}$ while the L55P-TTR-S-Hcy sample was populated of particles of larger $\mathrm{D}_{\mathrm{h}}$, corresponding to protein aggregates (Fig. 3C-E). Overall, these data suggest that, contrary to wt-TTR, S-homocysteinylation of L55P-TTR results in tetramer destabilization favouring the appearance of aggregation-prone low molecular weight species eventually resulting in the appearance of self-assembled large assemblies.

The FTIR spectra of both L55P-TTR-S-Hcy and L55P-TTR were found to be dominated by a component at $1630 \mathrm{~cm}-1$ and did not reveal any significant conformational change between the two samples. These data indicated that the S-homocysteinylation of L55P-TTR destabilized the tetrameric structure of the protein, confirming the $t$-Res assay. In addition, the absence of new peaks at around $1615 \mathrm{~cm}^{-1}$ or $1682 \mathrm{~cm}^{-1}$ (typical signatures of intermolecular $\beta$-sheets in protein aggregate) suggested that the S-homocysteinylation induced the appearance of aggregation-prone low molecular weight species without promoting amyloid formation (Fig. 3F). The tetrameric destabilization induced by Hcy presence is confirmed by Native- and SDS-PAGE of L55P-TTR (Fig. 3G).

\subsection{Conformational properties of homocysteinylated L55P-TTR}

We also analysed aggregation of L55P-TTR and L55P-TTR-S-Hcy by turbidity assay (Fig. 4A). At each incubation time, the L55P-TTR-S-Hcy complex displayed higher turbidity than L55P-TTR, suggesting that Shomocysteinylation increased the amount of higher molecular weight species in the sample. We also observed the morphology of L55P-TTR and L55P-TTR-S-Hcy samples by transmission electron microscopy (TEM). At each time (data not shown), after $96 \mathrm{~h}$ of incubation with Hcy the covalently modified TTR produced a larger amount of aggregated structures on the formvar dish than the unmodified protein (Fig. 4B).

\subsection{Hcy induces dissociation of L55P-TTR, protecting HL-1 cells from toxic effect of HypF oligomers}

It was previously reported that human cerebrospinal fluid (CSF) inhibited A 340 aggregation into amyloid fibrils in vitro [35] and that TTR was responsible of this effect due to its ability to form stable complexes with misfolded A $\beta 40$ [36]. Recently, it has been observed that TTR can suppress the toxicity of extracellularly added oligomers formed by two different peptides/proteins, A 442 and HypF-N [23,37]. In particular, an engineered, stable monomeric derivative of human TTR with intrinsic stability (M-TTR) engineered by introducing two methionine substitutions (F87 M and $\mathrm{L} 110 \mathrm{M}$ ) displayed disarranged subunit interfaces of the tetramer, which resulted in protection of SHSY5Y cells and rat primary neurons against oligomer-induced cytotoxicity [37]. Moreover, the interaction between HypF-N/Aß42 oligomers and M-TTR was found to involve oligomer clustering [36]. Based on these data we performed an MTT assay to assess the presence of monomeric species in the destabilized tetramer of L55P-TTR-S-Hcy sample. In particular, L55P-TTR $(5.0 \mu \mathrm{M})$ was incubated with homocysteine $(10 \mu \mathrm{M})$ for different lengths of time (30 min and96 h); then, each sample, was diluted to $1.2 \mu \mathrm{M}$ final concentrationin cultured cell medium containing $12 \mu \mathrm{M}$ HypF oligomers for $1 \mathrm{~h}$; murine cardiomyocytes (HL-1) were treated for $24 \mathrm{~h}$ with these samples; this cell line was chosen since it is susceptible to TTR amyloid cytotoxicity (Fig. 4D) [24]. Other cells were treated for $24 \mathrm{~h}$ with HypF oligomers, previously treated in cell medium without or with M-TTR $(1.2 \mu \mathrm{M})$. Fig. 4D shows that, under these conditions, L55P-TTR and L55P-TTR-S-Hcy were not toxic to HL-1 cells whereas about a 50 and $40 \%$ of reduction in cell viability upon exposure to HypF oligomers or M-TTR, respectively, was observed, in agreement with previous data [23]. However, when HL-1 cells were incubated with HypF oligomers previously treated with L55P-TTR, a $20 \%$ increase of viability was observed regardless of L55PTTR aging time; moreover, when the cells were incubated for $24 \mathrm{~h}$ with HypF oligomers pre-treated with L55P-TTR-S-Hcy aged $30 \mathrm{~min}$ or $96 \mathrm{~h}$, a $20 \%$ and a complete recovery of cell viability, respectively, was observed. A similar effect was seen also, when we used HypF oligomers pre-treated with M-TTR (Fig. 4D). This result indicates that L55P-TTRS-Hcy, particularly the L55P-TTR-S-Hcy $96 \mathrm{~h}$, behaves similarly to chaperonic M-TTR, suggesting that in the L55P-TTR-S-Hcy $96 \mathrm{~h}$ sample, a significant amount of monomeric TTR, sufficient to bind and to suppress HypF-N oligomers toxicity, is present.

\subsection{Homocysteinylation increases L55P cytotoxicity}

Once assessed the effect of S-homocysteinylation on L55P-TTR stability we tested L55P-TTR-S-Hcy cytotoxicity to HL-1 cells by the MTT assay. The cells were treated for $24 \mathrm{~h}$ with $5.0 \mu \mathrm{M}$ of L55P samples incubated in vitro for different times at $\mathrm{pH} 7.0$ in the presence or in the absence of Hcy. The MTT assay showed that all L55P-TTR samples obtained in the absence of Hcy induced a similar toxicity (about 10\%), whereas only the L55P-TTR-S-Hcy sample aggregated $96 \mathrm{~h}$ induced a significantly higher cell sufferance (about 35\%) (Fig. 5A). Confocal analysis on living cells using Fluo-3 AM, a dye that binds intracellular free $\mathrm{Ca}^{2+}$, showed that cell exposure to L55P-TTR incubated for $96 \mathrm{~h}$ with Hcy resulted in a significant and biphasic (peaks at $30 \mathrm{~min}$ and $60 \mathrm{~min}$ ) increase of intracellular free $\mathrm{Ca}^{2+}$, which may result in cytotoxic event as observed by the MTT assay results (Fig. 5 A, B, C).

\subsection{Effects of L55P-TTR and L55P-TTR-S-Hcy on kinematics contractile properties of cardiomyocyte syncytia}

We also sought to determine some functional correlates to the cytotoxic effects assessed by the MTT assay carried out on HL- 1 cells by checking the contractile properties of cardiac syncytia differentiated from mouse embryonic stem cells [31]. To this aim, the syncytia were exposed for $24 \mathrm{~h}$ to $5 \mu \mathrm{M}$ L55P-TTR or to L55P-TTR-S-Hcy aged $96 \mathrm{~h}$. On recorded AVI videos, chronotropic (beat frequency [Hz]), inotropic (contractility [pixel/s], contraction force $\left[\mathrm{pixel} / \mathrm{s}^{2}\right]$ ) and ergotropic (consumption of ATP for kinetic energy $\left[\mathrm{pixel}^{2} / \mathrm{s}^{2}\right.$ ]) features were mathematically calculated from the movement of the beating syncytia $[32,38]$. When compared to control cells, the analysis showed that differentiated cardiomyocytes exposed to L55P-TTR maintained unaltered ( $\mathrm{p}>0.05$ ) kinematics and dynamics properties. However, upon exposure to L55P-TTR-S-Hcy, the cardiomyocytes displayed a significant increase $(\mathrm{p}<0.05)$ of beat frequency, contraction force and kinetic energy (1.3, 1.3-, and 1.25-fold, respectively), while retaining substantially unaltered contractility (Fig. 6), in agreement with the increased intracellular $\mathrm{Ca}^{2+}$. These data are of interest; in fact, they suggest that functional correlates to the calcium-toxicity relation of aged L55P-Hcy do exist, supporting the idea that S-homocysteinylation of L55P-TTR resulting from hyperhomocysteinemia can be cardiotoxic in FAC and FAP.

\section{Discussion}

Both genetic and environmental factors contribute to the pathogenesis of complex diseases such as amyloid disease. The TTR amyloidosis include genetically determined pathologies such as familial amyloid polyneuropathy (FAP), including forms with heavy heart involvement (FAC), and the most represented sporadic forms with heart involvement (SSA). All these conditions are characterized by the presence of amyloid deposits in the heart and other tissues of TTR, either containing one out of a number of described mutations, or wild type, respectively; in particular, the L55P variant is mostly involved in cardiac dysfunction. Recent data have reported that the thiol group of 
A
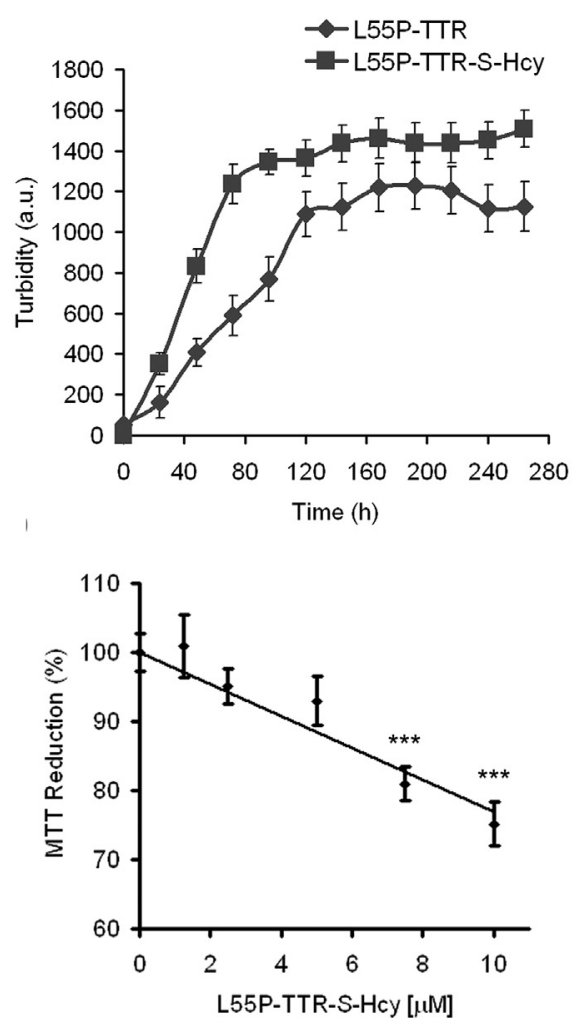

B
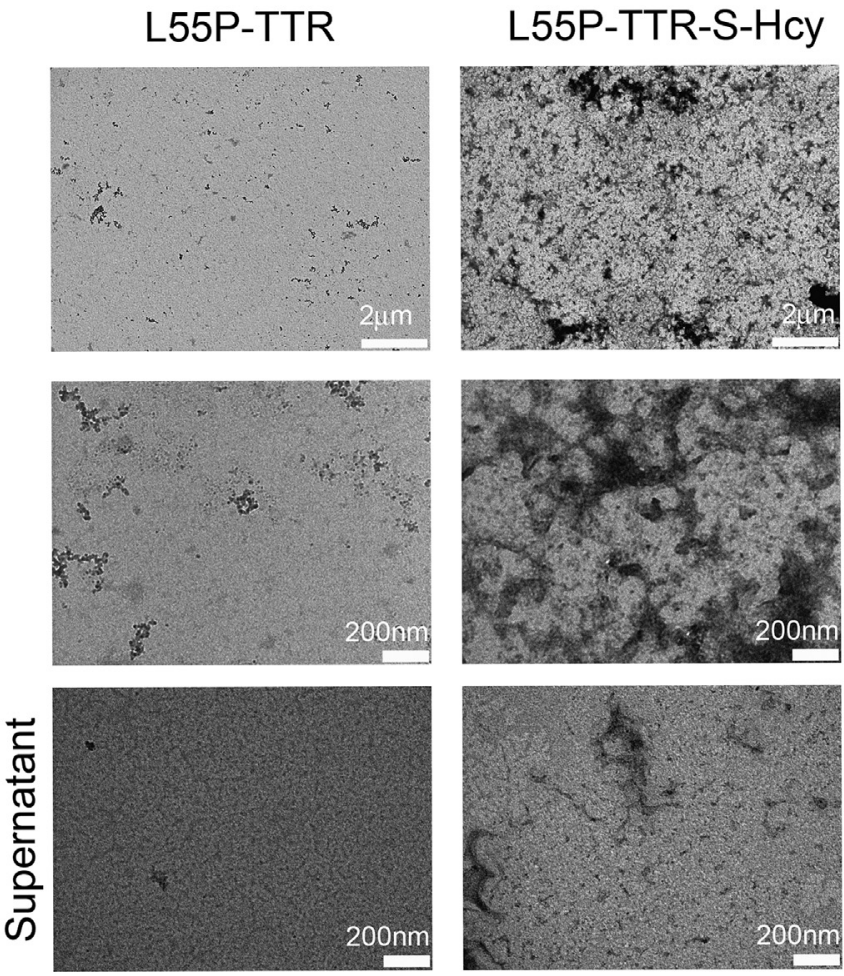
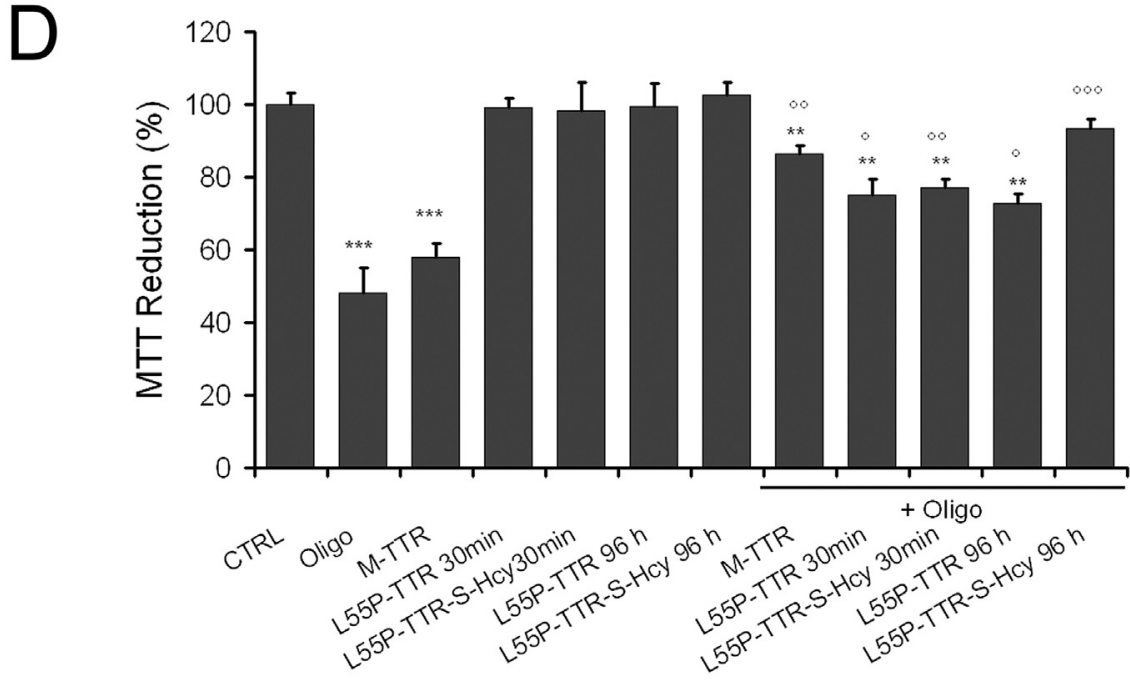

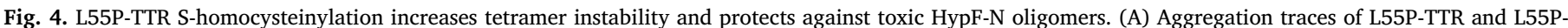

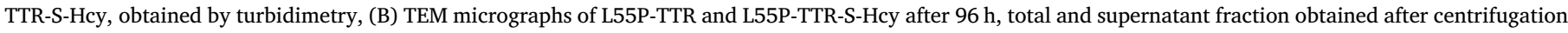

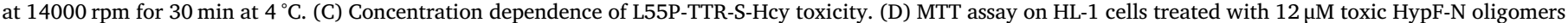

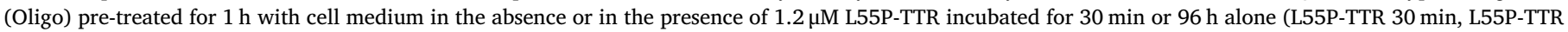

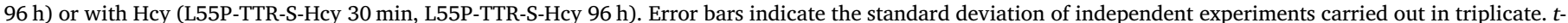

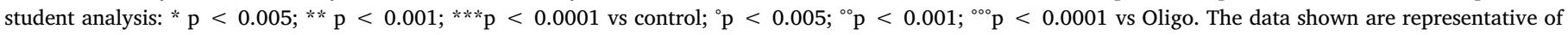
three independent experiments.

Cys $^{10}$ found in monomeric TTR is remarkably reactive and that its oxidation is involved in TTR tetramer destabilization [39,40]. These findings agree with the severe oxidative stress observed in FAP patients [14]. In addition to oxidation, other post-translational modification modify tetramer stability. For instance, S-cysteinylation of Cys ${ }^{10}$ destabilizes TTR [40,41], whereas S-sulfonation stabilizes TTR against aggregation under acidic conditions and at $\mathrm{pH} 9$ [41].

Hyperhomocysteinemia is considered as an independent risk factor for cardiovascular disease and an emerging risk factor for cognitive dysfunction in Alzheimer's disease [1]. Taking into account that high Hcy levels can initiate and speed atherosclerosis, that amyloid TTR accumulates in the heart, and that TTR can undergo S-homocysteinylation at its $\mathrm{Cys}^{10}$ residue both in vitro and in vivo [18], we investigated the effects of TTR S-homocysteinylation in terms of protein stabilization/destabilization, propensity to undergo amyloid aggregation and toxicity of the resulting aggregates. It has been reported that in 

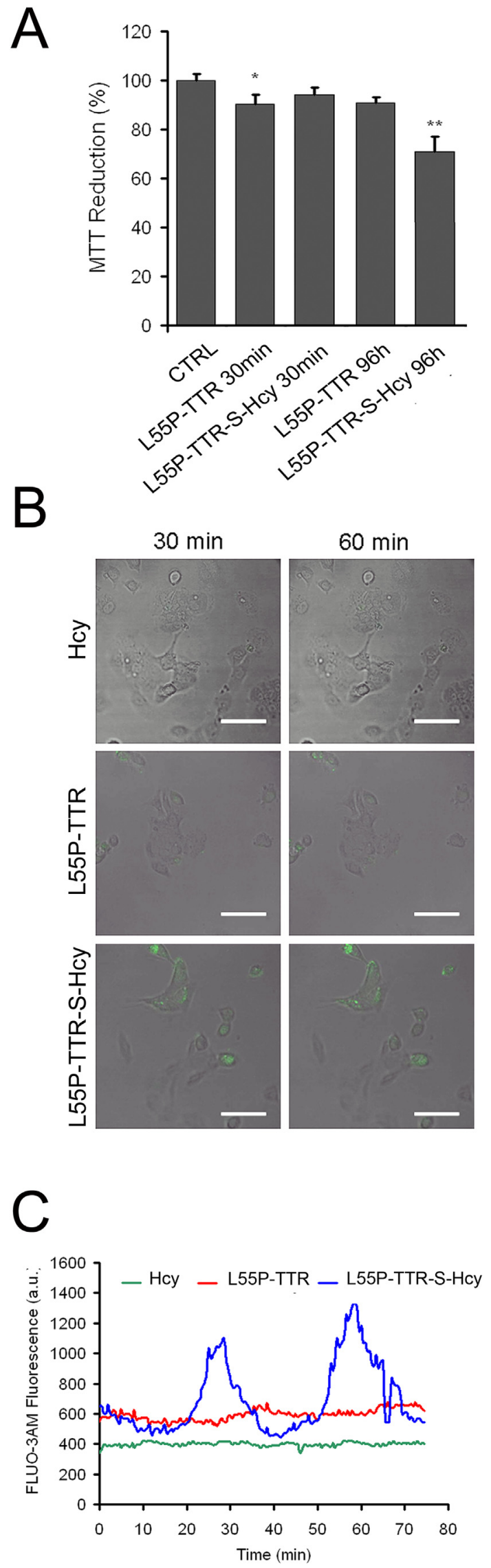

Fig. 5. Cytotoxicity of L55P-TTR-S-Hcy. (A) MTT assay on HL-1 cells treated with $5 \mu \mathrm{M}$ L55P-TTR incubated alone or with $10 \mu \mathrm{M}$ Hcy for $30 \mathrm{~min}$ or $96 \mathrm{~h}$. (B) Intracellular $\mathrm{Ca}^{2+}$ levels. (C) Time course of intracellular $\mathrm{Ca}^{2+}$ levels. Error bars indicate the standard deviation of independent experiments carried out in triplicate. t-student analysis: ${ }^{*} \mathrm{p}<0.005 ;{ }^{* *} \mathrm{p}<0.001 ;{ }^{* * *} \mathrm{p}<0.0001$.

patients affected by homocysteinuria, total homocysteine levels can easily raise up to $400-500 \mu \mathrm{M}$. However, in plasma of patients, about $70 \%$ of total Hcy is bound to protein cysteine residues via disulfidebond, and only $30 \%$ is in a free form $[42,43]$. Moreover, the "soluble fraction" of homocysteine includes homocystine, and different types of mixed disulfides including homocysteine-cysteine, and homocysteineglutathione, while the reduced free form of homocysteine does not exceed $2 \%$ of total homocysteine content [44]. This finding suggests that the fraction of homocysteine able to reacts with TTR is very low, raising the question whether TTR can realistically undergo homocysteinylation in vivo. Lim and co-workers reported that in a patient suffering end-stage renal disease, with total Hcy concentration of $20.7 \mu \mathrm{M}$, a very small amount of S-homocysteinylated TTR was detected [19]. Conversely, most of TTR isolated from plasma resulted S-homocysteinylated in homocystenuric patients showing a total Hcy concentration of $434 \mu \mathrm{M}$ [19]. Based on this evidence, we used $10 \mu \mathrm{M}$ reduced Hcy to perform our tests, confiding that such concentration was in the range of that detectable in patients affected by homocysteinuria [45].

The data presented in this study confirmed that TTR is S-homocysteinylated in vitro and this covalent modification stabilizes the tetrameric structure of wt-TTR while destabilizes the L55P-TTR tetramer, thereby favouring the accumulation of toxic monomeric species. Considering recent data indicating that monomeric TTR inhibits A $\beta$ peptide aggregation and amyloid plaque formation both in vitro and in vivo $[46,47]$, we also checked the presence of monomeric species arising from L55P-TTR S-homocysteinylation by analysing the residual chaperone ability of the S-homocysteinylated samples. Our in vitro analysis showed that monomeric L55P-TTR-S-Hcy reduces the cytotoxicity of preformed oligomers grown from HypF-N, similarly to the previously reported chaperonic activity of M-TTR [35,36]. Taken together, our findings suggest that S-homocysteinylation of L55P-TTR promotes the appearance of monomeric species with higher tendency to chaperone, as well as the M-TTR, toxic amyloid oligomers. In agreement with the different tetramer stability, native and S-homocysteinylated L55P-TTR exhibited different cytotoxicity to cultured cardiac cells. We found that the viability of cardiomyocytes exposed to L55P-TTR was affected by protein S-homocysteinylation only in the case of $96 \mathrm{~h}$-aged samples, whereas $30 \mathrm{~min}$-aged assemblies were harmless. Cytotoxicity also matched the intracellular $\mathrm{Ca}^{2+}$ levels in the same cells. In fact, we found significantly increased intracellular $\mathrm{Ca}^{2+}$ levels only in cells exposed to $96 \mathrm{~h}$-aged L55P-TTR-S-Hcy, further associating cell viability impairment to the rise of intracellular $\mathrm{Ca}^{2+}$. The toxic effects displayed by $96 \mathrm{~h}$-aged L55P-TTR-S-Hcy have functional consequences; in fact, when exposed to this sample, beating syncytia, obtained from the differentiation of R1 mouse embryonic stem cells showed altered kinematics and dynamics parameters. Beat frequency (chronotropic), contraction force (inotropic) and kinetic energy (ergotropic) were increased, possibly due to the impaired intracellular $\mathrm{Ca}^{2+}$ levels, whereas contractility, which represents the maximum contraction velocity, a parameter not expected to be heavily affected by $\mathrm{Ca}^{2+}$ levels, was unaltered. We did not check wt-TTR-S-Hcy cytotoxicity since we found that the chemical modification resulted in tetramer stabilization with maintenance of the native fold.

Different hypotheses have been proposed to describe the pathological features associated with hyperhomocysteinemia in humans; however, the cause of Hcy toxicity is not fully understood. In this sense, it has been proposed that accumulation of homocysteinylated proteins is an important risk factor for cardiovascular and neurodegenerative 


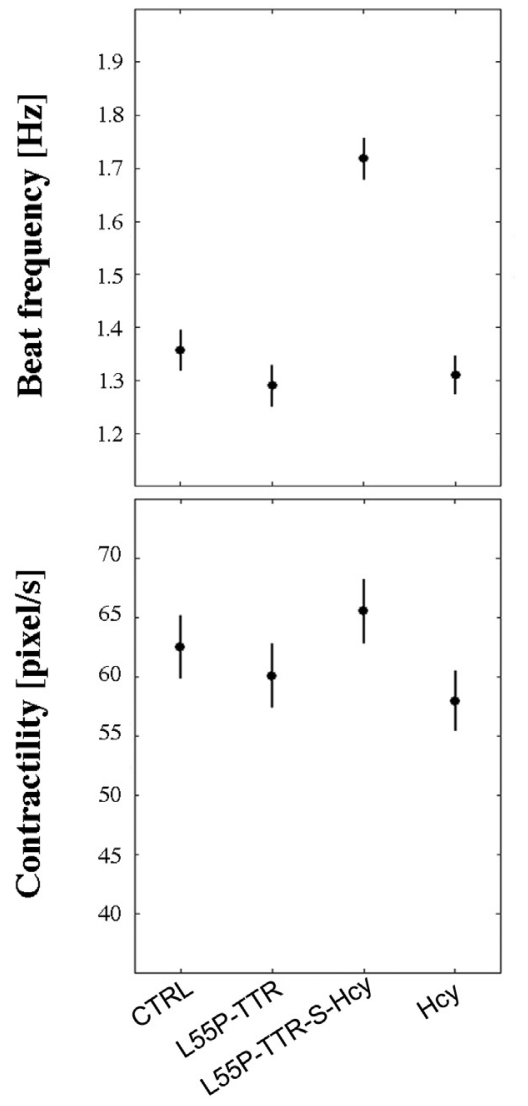

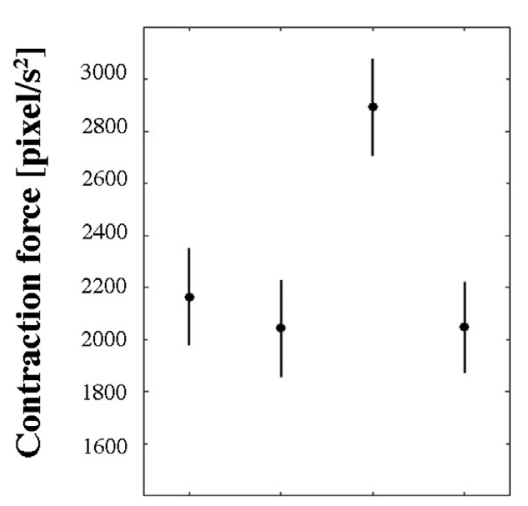

Fig. 6. Contractile properties of beating cardiac syncytia derived from embryonic stem cells differentiation. Contractile properties of beating syncytia exposed for $24 \mathrm{~h}$ to $5 \mu \mathrm{M}$ L55PTTR or L55P-TTR-S-Hcy ( $96 \mathrm{~h}$ ), compared to those recorded in unexposed control cells or in cells treated only with Hcy. Vertical bars represent the 95\% confidence intervals for the differences between means according to the Least Significant Difference statistical test. diseases. In this paper, we report that S-homocysteinylation can stabilize the native conformation of wt-TTR, while favouring destabilization of L55P-TTR that by itself is considered the causative agent of a form of early-onset familial amyloid polyneuropathy (FAP) and cardiomyopathy (FAC). Our results could have pathophysiological relevance, contributing to shed light into the molecular mechanisms underlying some pathological consequences described in patients affected by hyperhomocysteinemia.

\section{Acknowledgements}

This work has been supported by Ente Cassa di Risparmio di Firenze (grant n. 2015.1002A2202.3931). M.L. was supported by ANCC-COOP/ Airalzh ONLUS (Reg. n. 0043966.30-10- 359 2014) through the University of Florence (D.R.595/2016). This work has been supported by the Italian Ministry of Education, University and Research (MIUR): Dipartimenti di Eccellenza Program (2018-2022) - Dept. of Biology and Biotechnology "L. Spallanzani", University of Pavia (PR and SG). The authors wish to thank Prof. Fabrizio Chiti for providing HypF-N oligomers and M-TTR.

\section{References}

[1] R.F. de Bruijn, M.A. Ikram, Cardiovascular risk factors and future risk of Alzheimer's disease, BMC Med. 12 (2014) 130.

[2] Y. Silla, E. Sundaramoorthy, P. Talwar, S. Sengupta, S-linked protein homocysteinylation: identifying targets based on structural, physicochemical and protein-protein interactions of homocysteinylated proteins, Amino Acids 44 (5) (2013) 1307-1316.

[3] D.W. Jacobsen, O. Catanescu, P.M. Dibello, J.C. Barbato, Molecular targeting by homocysteine; a mechanism for vascular pathogenesis, Clin. Chem. Lab. Med. 43 (2005) 1076-1083.

[4] R. Rossi, D. Giustarini, A. Milzani, I. Dalle-Donne, Cysteinylation and homocysteinylation of plasma protein thiols during ageing of healthy human beings, $\mathrm{J}$. Cell. Mol. Med. 13 (9b) (2009) 3131-3140.

[5] F.P. Perez, J.I. Ilie, X. Zhou, D. Feinstein, D.A. Jurivich, Pathomolecular effects of homocysteine on the aging process: a new theory of aging, Med. Hypotheses 69
(2007) 149-160.

[6] R. Capasso, I. Sambri, A. Cimmino, S. Salemme, C. Lombardi, F. Acanfora, et al., Homocysteinylated albumin promotes increased monocyte-endothelial cell adhesion and up-regulation of MCP1, Hsp60 and ADAM17, PLoS One 7 (2) (2012) e31388.

[7] O. Roda, M.L. Valero, S. Peiro, D. Andreu, F.X. Real, P. Navarro, New insights into the tPA-annexin A2 interaction. Is annexin A2 CYS8 the sole requirement for this association? J. Biol. Chem. 278 (2003) 5702-5709.

[8] A. Undas, E.B. Williams, S. Butenas, T. Orfeo, K.G. Mann, Homocysteine inhibits inactivation of factor Va by activated protein C, J. Biol. Chem. 276 (2001) 4389-4397.

[9] A. Bescond, T. Augier, C. Chareyre, D. Garçon, W. Hornebeck, P. Charpiot, Influence of homocysteine on matrix metalloproteinase-2: activation and activity, Biochem. Biophys. Res. Commun. 263 (1999) 498-503.

[10] A.K. Majors, S. Sengupta, B. Willard, M.T. Kinter, R.E. Pyeritz, D.W. Jacobsen, Homocysteine binds to human plasma fibronectin and inhibits its interaction with fibrin, Arterioscler. Thromb. Vasc. Biol. 22 (2002) 1354-1359.

[11] A. Zinellu, S. Sotgia, E. Pisanu, G. Loriga, L. Deiana, A.E. Satta, C. Carru, LDL S homocysteinylation decrease in chronic kidney disease patients undergone lipid lowering therapy, Eur. J. Pharm. Sci. 47 (1) (2012) 117-123.

[12] J.C. Barbato, O. Catanescu, K. Murray, P.M. DiBello, D.W. Jacobsen, Targeting of metallothionein by L-homocysteine: a novel mechanism for disruption of zinc and redox homeostasis, Arterioscler. Thromb. Vasc. Biol. 27 (2007) 49-54.

[13] J.T. White, J.W. Kelly, Support for the multigenic hypothesis of amyloidosis: the binding stoichiometry of retinol-binding protein, vitamin A, and thyroid hormone influences transthyretin amyloidogenicity in vitro, Proc. Natl. Acad. Sci. U. S. A. 98 (2001) 13019-13024.

[14] Y. Ando, M. Nakamura, S. Araki, Transthyretin-related familial amyloidotic polyneuropathy, Arch. Neurol. 62 (2005) 1057-1062.

[15] C. Rapezzi, G. Merlini, C.C. Quarta, L. Riva, S. Longhi, O. Leone, et al., Systemic cardiac amyloidoses: disease profiles and clinical courses of the 3 main types, Circulation 120 (2009) 1203-1212.

[16] A. Quintas, M.J. Saraiva, R.M. Brito, The amyloidogenic potential of transthyretin variants correlates with their tendency to aggregate in solution, FEBS Lett. 418 (1997) 297-300.

[17] H.A. Lashuel, Z. Lai, J.W. Kelly, Characterization of the transthyretin acid denaturation pathways by analytical ultracentrifugation: implications for wild-type, V30M, and L55P amyloid fibril formation, Biochemistry 37 (51) (1998) 17851-17864.

[18] N. Hanyu, T. Shimizu, K. Yamauchi, N. Okumura, H. Hidaka, Characterization of cysteine and homocysteine bound to human serum transthyretin, Clin. Chim. Acta 403 (2009) 70-75.

[19] A. Lim, S. Sengupta, M.E. McComb, R. Théberge, W.G. Wilson, C.E. Costello, D.W. Jacobsen, In vitro and in vivo interactions of homocysteine with human 
plasma transthyretin, JBC 278 (2003) 49707-49713.

[20] J.O. Sass, T. Nakanishi, T. Sato, A. Shimizu, S-Homocysteinylation of transthyretin is detected in plasma and serum of humans with different types of Hyperhomocysteinemia, Biochem. Biophys. Res. Commun. 310 (1) (2003) 242-246.

[21] P.P. Mangione, R. Porcari, J.D. Gillmore, P. Pucci, M. Monti, M. Porcari, et al., Proteolytic cleavage of Ser52Pro variant transthyretin triggers its amyloid fibrillogenesis, Proc. Natl. Acad. Sci. U. S. A. 111 (2014) 1539-1544.

[22] S. Campioni, M.F. Mossuto, S. Torrassa, G. Calloni, P.P. de Laureto, A. Relini, et al., Conformational properties of the aggregation precursor state of HypF-N, J. Mol. Biol. 379 (3) (2008) 554-567.

[23] S. Cappelli, A. Penco, B. Mannini, R. Cascella, M.R. Wilson, H. Ecroyd, et al., Effect of molecular chaperones on aberrant protein oligomers in vitro: super-versus substoichiometric chaperone concentrations, Biol. Chem. 397 (5) (2016) 401-415.

[24] L. Sartiani, M. Bucciantini, V. Spinelli, M. Leri, A. Natalello, D. Nosi, et al., Biochemical and electrophysiological modification of amyloid transthyretin on cardiomyocytes, Biophys. J. 111 (9) (2016) 2024-2038.

[25] D. Ami, P. Mereghetti, M. Leri, S. Giorgetti, A. Natalello, S.M. Doglia, et al., A FTIR microspectroscopy study of the structural and biochemical perturbations induced by natively folded and aggregated transthyretin in HL-1 cardiomyocytes, Sci. Rep. 8 (1) (2018) 12508 .

[26] G. Arsequell, A. Planas, Methods to evaluate the inhibition of TTR fibrillogenesis induced by small ligands, Curr. Med. Chem. 19 (15) (2012) 2343-2355.

[27] P. Florio, C. Folli, M. Cianci, D. Del Rio, G. Zanotti, R. Berni, Transthyretin binding heterogeneity and anti-amyloidogenic activity of natural polyphenols and their metabolites, J. Biol. Chem. 290 (50) (2015) 29769-29780.

[28] S.M. White, P.E. Constantin, W.C. Claycomb, Cardiac physiology at the cellular level: use of cultured HL-1 cardiomyocytes for studies of cardiac muscle cell structure and function, Am. J. Physiol. Heart Circ. Physiol. 286 (2004) H823-H829.

[29] P. Rebuzzini, D. Pignalosa, G. Mazzini, R. Di Liberto, A. Coppola, N. Terranova, et al., Mouse embryonic stem cells that survive $\gamma$-rays exposure maintain pluripotent differentiation potential and genome stability, J. Cell. Physiol. 227 (3) (2012) 1242-1249.

[30] K.R. Boheler, J. Czyz, D. Tweedie, H.T. Yang, S.V. Anisimov, A.M. Wobus, Differentiation of pluripotent embryonic stem cells into cardiomyocytes, Circ. Res. 91 (3) (2002) 189-201 (Review).

[31] P. Rebuzzini, E. Cebral, L. Fassina, C.A. Redi, M. Zuccotti, S. Garagna, Arsenic trioxide alters the differentiation of mouse embryonic stem cell into cardiomyocytes, Sci. Rep. 5 (2015) 14993.

[32] L. Fassina, A. Di Grazia, F. Naro, L. Monaco, M.G. De Angelis, G. Magenes, Video evaluation of the kinematics and dynamics of the beating cardiac syncytium: an alternative to the Langendorff method, Int. J. Artif Organs. 34 (7) (2011) 546-558.

[33] H. Refsum, E. Nurk, A.D. Smith, P.M. Ueland, C.G. Gjesdal, I. Bjelland, et al., The
Hordaland homocysteine study: a community-based study of homocysteine, its determinants, and associations with disease, J. Nutr. 136 (2006) 1731S-1740S.

[34] R.H. Pires, Á. Karsai, M.J. Saraiva, A.M. Damas, M.S. Kellermayer, Distinct annular oligomers captured along the assembly and disassembly pathways of transthyretin amyloid protofibrils, PLoS One 7 (2012) e44992.

[35] N. Lu, Q. Yang, J. Li, R. Tian, Y.Y. Peng, Inhibitory effect of human serum albumin on Cu-induced A $\beta(40)$ aggregation and toxicity, Eur. J. Pharmacol. 767 (2015) $160-164$.

[36] X. Li, X. Zhang, A.R. Ladiwala, D. Du, J.K. Yadav, et al., Mechanisms of transthyretin inhibition of $\beta$-amyloid aggregation in vitro, J. Neurosci. 33 (50) (2013) 19423-19433.

[37] R. Cascella, S. Conti, B. Mannini, X. Li, J.N. Buxbaum, B. Tiribilli, et al., Transthyretin suppresses the toxicity of oligomers formed by misfolded proteins in vitro, Biochim. Biophys. Acta 1832 (2013) 2302-2314.

[38] P. Rebuzzini, L. Fassina, F. Mulas, R. Bellazzi, C.A. Redi, R. Di Liberto, et al., Mouse embryonic stem cells irradiated with $\gamma$-rays differentiate into cardiomyocytes but with altered contractile properties, Mutat. Res. 756 (1-2) (2013) 37-45.

[39] L. Zhao, J.N. Buxbaum, N. Reixach, Age-related oxidative modifications of transthyretin modulate its amyloidogenicity, Biochemistry 52 (11) (2013) 1913-1926.

[40] J.S. Kingsbury, T.M. Laue, E.S. Klimtchuk, R. Théberge, C.E. Costello, L.H. Connors, The modulation of transthyretin tetramer stability by cysteine 10 adducts and the drug diflunisal. Direct analysis by fluorescence-detected analytical ultracentrifugation, J. Biol. Chem. 283 (18) (2008) 11887-11896.

[41] Q. Zhang, J.W. Kelly, Cys10 mixed disulfides make transthyretin more amyloidogenic under mildly acidic conditions, Biochemistry 42 (29) (2003) 8756-8761.

[42] K.J. Rudreshkumar, V. Majumdar, D. Nagaraja, R. Christopher, Relevance of plasma levels of free homocysteine and methionine as risk predictors for ischemic stroke in the young, Clin. Nutr. 37 (5) (2018) 1715-1721.

[43] M.G. van Oijen, B.E. Claessen, N. Clappers, A. van Schaik, R.J. Laheij, J.B. Jansen, et al., Prognostic value of free plasma homocysteine levels in patients hospitalized with acute coronary syndrome, Am. J. Cardiol. 102 (2) (2008) 135-139.

[44] A.V. Glushchenko, D.W. Jacobsen, Molecular targeting of proteins by L-homocysteine: mechanistic implications for vascular disease, Antioxid. Redox Signal. 9 (11) (2007) 1883-1898 (Review).

[45] M.A. Mansoor, P.M. Ueland, A. Aarsland, A.M. Svardal, Redox status and protein binding of plasma homocysteine and other aminothiols in patients with homocystinuria, Metabolism 42 (1993) 1481-1485.

[46] A.L. Schwarzman, L. Gregori, M.P. Vitek, S. Lyubski, W.J. Strittmatter, J.J. Enghilde, et al., Transthyretin sequesters amyloid beta protein and prevents amyloid formation, Proc. Natl. Acad. Sci. U. S. A. 91 (18) (1994) 8368-8372.

[47] J.N. Buxbaum, Z. Ye, N. Reixach, L. Friske, C. Levy, P. Das, et al., Transthyretin protects Alzheimer's mice from the behavioral and biochemical effects of Abeta toxicity, Proc. Natl. Acad. Sci. U. S. A. 105 (7) (2008) 2681-2686. 